\title{
Moment Closure Hierarchies for the Boltzmann-Poisson Equation
}

\author{
C. DAVID LEVERMORE* \\ Department of Mathematics, University of Arizona, Tucson, AZ 85721, USA
}

\begin{abstract}
We outline a systematic nonperturbative derivation of a hierarchy of closed systems of moment equations that can be applied to any kinetic description of electrons in a semiconductor. This entropy based closure procedure extends one that was introduced in the context of gas dynamics. In the context of semiconductors, this procedure yields generalizations of socalled hydrodynamic models. It is illustrated on the semiclassical Boltzmann-Poisson equation for a single conduction band in the parabolic band approximation.
\end{abstract}

Keywords: Boltzmann-Poisson, drift-diffusion, entropy, hydrodynamic models, hyperbolicity, moment closures

\section{INTRODUCTION}

An entropy based procedure has been proposed in [4] to construct systems of moment equations from any kinetic description of an isolated physical system. Here we extend this procedure to physical systems in contact with a thermal bath, such as electrons in a semiconductor. The resulting equations properly capture macroscopic regimes in the limit of small mean free paths, while yielding physically reasonable values for physical fluxes (and other quantities) in transition regimes. Such models may provide a bridge over the transition regime that may be useful in the construction of hybrid macroscopic/kinetic simulations.

The closure procedure can be applied to a general class of kinetic theories but is herein illustrated on the semiclassical Boltzmann-Poisson equation for a single conduction band using the parabolic band approximation. The velocity of a carrier electron is therefore related to its wave number $k$ by $v=\hbar k / m_{e}$, where $m_{e}$ is the effective electron mass. Specifically, we consider $F=F(t, x, v)$, a carrier electron distribution function of time $t$, position $x$ and velocity $v$ that is governed by

$$
\partial_{t} F+v \cdot \nabla_{x} F-\frac{q_{e}}{m_{e}} E \cdot \nabla_{v} F=C(F) .
$$

Here $q_{e}$ is the magnitude of the electron charge and $E=-\nabla_{x} \Phi$ is the electric field, which is expressed in terms of the electric potential $\Phi=\Phi(x, t)$ that satisfies

$$
-\nabla_{x} \cdot\left[\varepsilon_{d} \nabla_{x} \Phi\right]=q_{e}\left(Z_{d}-\langle F\rangle\right)
$$

where $\varepsilon_{d}=\varepsilon_{d}(x)$ is the permittivity of the device, $Z_{d}=$ $Z_{d}(x)$ is the charge density of the donor and acceptor ions, and $(F)$ is the density of carrier electrons. Here we have introduced the shorthand $\langle\cdot\rangle$ to denote integration over $v$ : hence, for any function $f=f(v)$ one has

$$
\langle f\rangle \equiv \int_{\mathbb{R}^{3}} f(v) d^{3} v
$$

* Tel: 520-621-4397. Fax: 520-621-8322.E-mail: 1vrmr@math.arizona.edu 
The collision operator $C$ models the interaction of the electrons with background lattice impurities, phonons, and other electrons. It is assumed to act only on the $v$ variable of $F$. The electrons are assumed to be in contact with a thermal bath of lattice impurities and phonons at a constant temperature $\theta_{\ell}\left(=k_{B} T_{\ell}\right)$. Moreover, the electrons will be described by either Maxwell-Boltzmann or Fermi-Dirac statistics, and the entropy integrand $\eta(f)$ for the collision operator $C$ will given respectively by

$$
\begin{aligned}
& \eta_{M B}(f) \equiv f \log f-f+\frac{m_{e}}{2 \theta_{\ell}}|v|^{2} f \\
& \eta_{F D}(f) \equiv f \log f+(1-f) \log (1-f)+\frac{m_{e}}{2 \theta_{\ell}}|v|^{2} f .
\end{aligned}
$$

In either case it is assumed that

$$
\left\langle\partial_{f} \eta(f) C(f)\right\rangle \leq 0 \quad \text { for every } f
$$

and that for every $f$ the following are equivalent:

$$
\begin{aligned}
& \text { (i) }\left\langle\partial_{f} \eta(f) C(f)\right\rangle=0, \quad(\text { ii) } C(f)=0, \\
& \text { (iii) } \partial_{f} \eta(f)=\beta \text { for some } \beta \in \mathbb{R} \text {. }
\end{aligned}
$$

For example, collision operators that model the lattice impurity and phonon interactions which are consistent with either Maxwell-Boltzmann or Fermi-Dirac statistics [6] are respectively

$$
\begin{aligned}
C_{M B}(F)=\int_{\mathbb{R}^{3}} S_{\ell}\left(v, v^{\prime}\right) & {\left[M_{\ell} F^{\prime}-M_{\ell}^{\prime} F\right] d^{3} v^{\prime}, } \\
C_{F D}(F)=\int_{\mathbb{R}^{3}} S_{\ell}\left(v, v^{\prime}\right) & {\left[M_{\ell} F^{\prime}(1-F)\right.} \\
& \left.-M_{\ell}^{\prime} F\left(1-F^{\prime}\right)\right] d^{3} v^{\prime},
\end{aligned}
$$

where $M_{\ell}=M_{\ell}(v)$ is the Maxwellian at the lattice temperature $\theta_{\ell}$ that is given by

$$
M_{\ell}(v) \equiv \frac{1}{\left(2 \pi \theta_{\ell}\right)^{3 / 2}} \exp \left(-\frac{m_{e}}{2 \theta_{\ell}}|v|^{2}\right),
$$

and $S_{\ell}\left(v, v^{\prime}\right)=S_{\ell}\left(v^{\prime}, v\right)>0$ is the transition kernel for the lattice-electron interactions. A prime on a $M_{\ell}$ or $F$ in (1.7) indicates the velocity argument is to be evaluated at $v$ ' rather than $v$.

These properties lead to a local dissipation law that is satisfied by every solution $F=F(t, x, v)$ of the Boltzmann-Poisson system (1.1-1.2) and which takes the form

$$
\begin{array}{r}
\partial_{t}\left(\langle\eta(F)\rangle+\frac{\varepsilon_{d}}{2 \theta_{\ell}}\left|\nabla_{x} \Phi\right|^{2}\right) \\
+\nabla_{x} \cdot\left[\langle v \eta(F)\rangle-\frac{\varepsilon_{d}}{\theta_{\ell}} \Phi \partial_{t} \nabla_{x} \Phi-\frac{q_{e}}{\theta_{\ell}} \Phi\langle v F\rangle\right] \\
=\left\langle\partial_{F} \eta(F) C(F)\right\rangle \leq 0,
\end{array}
$$

Here $\langle(\eta(F)\rangle$ and $\langle v \eta(F)\rangle$ are called respectively the electron entropy density and flux, while $\left\langle\partial_{F} \eta(F) C(F)\right\rangle$ is called the entropy dissipation rate.

Equation (iii) of (1.6) can be solved for the local equilibria in terms of the Legendre transform of $\eta$, which is the convex function $\eta^{*}=\eta^{*}(y)$ that is defined implicitly through the relations

$$
\eta^{*}(y)+\eta(z)=y z, \quad y=\partial_{z} \eta(z) .
$$

Specifically, for the entropy integrands given by (1.4) one finds that

$$
\eta_{M B}^{*}(y)=M_{\ell} e^{y}, \quad \eta_{F D}^{*}(y)=\log \left(1+M_{\ell} e^{y}\right) .
$$

It is easy to verify the dual nature of this transformation from (1.10) by using implicit differentiation to check that $z=\partial_{y} \eta^{*}(y)$; it is then clear from (1.10) that the Legendre transform of $\eta^{*}$ is again $\eta$. Hence, relation (iii) is equivalent to

$$
\text { (iv) } f=\partial_{y} \eta^{*}(\beta) \text { for some } \beta \in \mathbb{R} \text {. }
$$

This yields the usual Maxwell-Boltzmann or FremiDirac equilibria for the corresponding $\eta *$ given by (1.11).

\section{CLOSURE PROCEDURE}

In general, the development of moment equations begins with the choice of a finite dimensional linear subspace $\mathbb{M}$ of functions of $v$ (here chosen to be polynomials). Let $M$ be the dimension of this space and $\left\{m_{i}=m_{i}(v)\right\}_{i=1}^{M}$ be a basis. Denote the column $M$ vector of these basis elements by $\boldsymbol{m}=\boldsymbol{m}(v)$, so that every $m \in \mathbb{M}$ has a unique representation in the form $m(v)=\alpha^{T} \boldsymbol{m}(v)$ for some $\alpha \in \mathbb{R}^{M}$, where $\boldsymbol{\alpha}^{T}$ is the matrix transpose of the column vector $\boldsymbol{\alpha}$. Now taking the moments of the Boltzmann-Poisson equation (1.1) over the vector $m(v)$ leads to the system

$$
\partial_{t}\langle\mathbf{m} F\rangle+\nabla_{x} \cdot\langle v \mathbf{m} F\rangle+\frac{q_{e}}{m_{e}} E \cdot\left\langle\left(\nabla_{v} \mathbf{m}\right) F\right\rangle=\langle\mathbf{m} C(F)\rangle .
$$

It must be noted that it is not known whether the quantities appearing in this equation are well defined functions for every solution $F$ of the Boltzmann-Poison equation. Here we shall suppose that these quantities are well defined and consider system (2.1) 
formally. The so-called "moment closure problem" is then to express the above densities $\langle\boldsymbol{m} F\rangle$, fluxes $\langle v \boldsymbol{m} F\rangle$, lower order terms $\left\langle\left(\nabla_{v} \boldsymbol{m}\right) F\right\rangle$, and collisional terms $\langle\boldsymbol{m} C(F)\rangle$ as a function of $M$ variables (traditionally the densities themselves [3]), thus formally closing the system.

The closure proceeds in two steps. The first ensures that every member of the hierarchy is hyperbolic and has an entropy. This implies that, unlike for many traditional approaches, the resulting equations are formally well-posed. The second step involves modifying the collisional terms so that each member of the hierarchy recovers the proper drift-diffusion approximation. This is done through the introduction of generalized BGK type collision operators. Here we will outline only the first step; all the details of both steps as well as discussions of boundary conditions and numerical methods are presented in [5].

Some of the aforementioned objectives can be achieved in part by requiring that $M$ satisfy:

(I) $\mathbb{D} \equiv \operatorname{span}\{1, v\} \subset \mathbb{M}$

(II) $\mathrm{M}$ is invariant under rotation and translation.

The span notation introduced here in the definition of $\mathbb{D}$ is generally applied to a collection of scalars, vectors, and tensors and means all scalar-valued linear combinations of their components. In particular, $\mathbb{D}$ consists of all linear combinations of the scalar 1 and the components of the vector $v$. Condition (I) above is the minimal requirement needed to recover the proper equilibrium and to evaluate the fluxes, while condition (II) is needed to evaluate the lower order terms because translation invariance implies $M$ is closed under the action of $\nabla_{v}$.

The moment closure prescribed here places an additional requirement on the linear subspace $\mathbb{M}$. Given any $\mathbb{M}$, this requirement is formulated in terms the associated subset $\mathbb{M}_{c} \equiv\left\{m \in \mathbb{M}:\left\langle\eta^{*}(m)\right\rangle<\infty\right\}$. The convexity of $\eta^{*}$ ensures that $\mathbb{M}_{c}$ is a convex cone in $\mathbb{M}$. We will construct a self-consistent closure of the Boltzmann-Poisson equation (1.1) for each $\mathbb{M}$ that satisfies conditions (I), (II), and also:

(III) the cone $\mathbb{M}_{c}$ has a nonempty interior in $\mathbb{M}$.
We shall call such subspaces admissible and all other subspaces inadmissible. Because $\mathbb{M}_{c}$ only contains polynomials $m(v)$ such that $\eta^{*}(m(v)) \rightarrow 0$ as $|v| \rightarrow \infty$, with the exception of $\mathbb{D}$, condition (III) of (2.3) can only be met by linear spaces of polynomials over $v$ with even maximal degree. In general, a linear space of even maximal degree that is invariant under rotations and translations and contains $\mathbb{D}$ can be admissible, but might not be. A general discussion of admissible spaces can be found in [4].

The closure procedure may now be stated simply. Let $F$ in the moment system (2.1) have the form

$$
F=\mathcal{M}(\boldsymbol{\alpha}) \equiv \partial_{y} \eta^{*}\left(\boldsymbol{\alpha}^{T} \mathbf{m}(v)\right)
$$

where $\boldsymbol{\alpha}^{T}$ is the transpose of some $\boldsymbol{\alpha}=\boldsymbol{\alpha}(t, x)$ with values in $\mathbb{R}^{M}$ such that $\boldsymbol{\alpha}^{T} \boldsymbol{m}(v)$ lies in the cone $\mathbb{M}_{c}$. This results in the system of $M$ equations (2.1) for the $M$ unknowns $\alpha$ given by

$$
\begin{aligned}
\partial_{t}\langle\mathbf{m} \mathcal{M}(\boldsymbol{\alpha})\rangle+\nabla_{x} \cdot\langle v \mathbf{m} \mathcal{M}(\boldsymbol{\alpha})\rangle \\
\quad+\frac{q_{e}}{m_{e}} E \cdot\left\langle\left(\nabla_{v} \mathbf{m}\right) \mathcal{M}(\boldsymbol{\alpha})\right\rangle=\langle\mathbf{m} \mathcal{C}(\mathcal{M}(\boldsymbol{\alpha}))\rangle
\end{aligned}
$$

where the moment densities, moment fluxes, lower order terms, and collisional terms are expressed in terms of $\alpha$ as the integrals $\langle\boldsymbol{m} \mathcal{M}(\boldsymbol{\alpha})\rangle,\langle\cup \boldsymbol{m} \mathcal{M}(\boldsymbol{\alpha})\rangle$, $\left\langle\left(\nabla_{v} \boldsymbol{m}\right) \mathcal{M}(\boldsymbol{\alpha})\right\rangle$ and $\langle\boldsymbol{m} C(\mathcal{M}(\boldsymbol{\alpha}))\rangle$. Each admissible space $\mathbb{M}$ generates such a closure, and the hierarchy of such closures is partially ordered by the relation $\subset$ acting on the admissible spaces. For the admissible space $\mathbb{M}=$ $\operatorname{span}\left\{1, v,|v|^{2}\right\}$ with Maxwell-Boltzmann statistics, the form of $F$ given by (2.4) will be that of a shifted (drifted) Maxwellian [6] while the moment system (2.5) will becomes a "hydrodynamic" model [5].

The moment system (2.5) has remarkable structural features that make it highly attractive theoretically and that facilitate its practical implementation. This structure can be brought out by recasting the moment system (2.5) in terms of the density potential $h^{*}$ and the flux potential $j^{*}$ defined by

$$
h^{*}(\boldsymbol{\alpha}) \equiv\left\langle\eta^{*}\left(\boldsymbol{\alpha}^{T} \mathbf{m}\right)\right\rangle, \quad j^{*}(\boldsymbol{\alpha}) \equiv\left\langle\eta^{*}\left(\boldsymbol{\alpha}^{T} \mathbf{m}\right)\right\rangle,
$$

and the vectors of lower order terms $\boldsymbol{l}^{*}$ and collisional relaxation terms $r^{*}$ defined by 
$\mathbf{l}^{*}(\boldsymbol{\alpha}) \equiv\left\langle\left(\nabla_{v} \mathbf{m}\right) \mathcal{M}(\boldsymbol{\alpha})\right\rangle, \quad \mathbf{r}^{*}(\boldsymbol{\alpha}) \equiv\langle(\mathbf{m} \mathcal{C}(\mathcal{M}(\boldsymbol{\alpha}))\rangle$

Differentiating (2.6) with respect to $\boldsymbol{\alpha}$ using (2.4) yields the relations

$h_{\boldsymbol{\alpha}}^{*}(\boldsymbol{\alpha})=\langle\mathbf{m} \mathcal{M}(\boldsymbol{\alpha})\rangle, \quad j_{\boldsymbol{\alpha}}^{*}(\boldsymbol{\alpha})=\langle v \mathbf{m} \mathcal{M}(\boldsymbol{\alpha})\rangle$,

where the right sides are just the moment densities and fluxes that appear in (2.5). The closed moment system (2.5) can therefore be put into the so-called potential form of Godunov [2]

$\partial_{t} h_{\boldsymbol{\alpha}}^{*}(\boldsymbol{\alpha})+\nabla_{x} \cdot j_{\boldsymbol{\alpha}}^{*}(\boldsymbol{\alpha})+\frac{q_{e}}{m_{e}} E \cdot \mathbf{l}^{*}(\boldsymbol{\alpha})=\mathbf{r}^{*}(\boldsymbol{\alpha})$.

Moreover, because each component of $v$ and $\nabla_{v} \boldsymbol{m}$ is in $\mathbb{M}$ each component of either the flux potential $j^{*}$ and the lower order terms $l *$ is itself a moment density and hence expressible as a derivative of the density potential $h^{*}$. Therefore, evaluating the left side of (2.9) requires only a twice differentiable evaluation of the scalar function $h^{*}$ in terms of $\boldsymbol{\alpha}$. Of course, such an evaluation of $h^{*}$ is generally going to be quite complicated, but this is the price to be paid for the structural simplicity that emerges $[4,5]$.

The moment system (2.5) has many desirable properties that can be read off from its potential form (2.9), many of which are lacking in traditional closures. The most important of these is its hyperbolicity, which ensures that the system is at least linearly wellposed. This property follows from the fact that the density potential $h^{*}=h^{*}(\boldsymbol{\alpha})$ is a strictly convex function with a positive definite Hessian matrix

$$
h_{\boldsymbol{\alpha} \boldsymbol{\alpha}}^{*}(\boldsymbol{\alpha})=\left\langle\mathbf{m m}^{T} \mathcal{M}(\boldsymbol{\alpha})\right\rangle .
$$

The hyperbolicity of (2.9) then becomes evident upon rewriting it in the form

$$
h_{\boldsymbol{\alpha} \boldsymbol{\alpha}}^{*}(\boldsymbol{\alpha}) \partial_{t} \boldsymbol{\alpha}+j_{\boldsymbol{\alpha} \boldsymbol{\alpha}}^{*}(\boldsymbol{\alpha}) \cdot \nabla_{x} \boldsymbol{\alpha}+\frac{q_{e}}{m_{e}} E \cdot \mathbf{l}^{*}(\boldsymbol{\alpha})=\mathbf{r}^{*}(\boldsymbol{\alpha}) .
$$

By the positive definiteness of $h^{*}{ }_{\alpha \alpha}$ and the symmetry of $j^{*}{ }_{\alpha \alpha}$, this has the Godunov form of a symmetric hyperbolic system for $\boldsymbol{\alpha}[2]$.

Another important property of the moment system (2.5) that is exposed by the its potential form (2.9) is the existence of an entropy that is locally dissipated.
Indeed, by multiplying (2.11) on the left by $\boldsymbol{\alpha}^{T}$ and using the Poisson equation (1.2) one obtains

$$
\begin{aligned}
& \partial_{t}\left(\boldsymbol{\alpha}^{T} h_{\boldsymbol{\alpha}}^{*}(\boldsymbol{\alpha})-h^{*}(\boldsymbol{\alpha})+x \frac{\varepsilon_{d}}{2 \theta_{\ell}}\left|\nabla_{x} \Phi\right|^{2}\right) \\
& +\nabla_{x} \cdot\left[\boldsymbol{\alpha}^{T} j_{\boldsymbol{\alpha}}^{*}(\boldsymbol{\alpha})-j^{*}(\boldsymbol{\alpha})-\frac{\varepsilon_{d}}{\theta_{\ell}} \Phi \partial_{t} \nabla_{x} \Phi\right. \\
& \left.-\frac{q_{e}}{\theta_{\ell}} \Phi\langle v \mathcal{M}(\boldsymbol{\alpha})\rangle\right]=\boldsymbol{\alpha}^{T} \mathbf{r}^{*}(\boldsymbol{\alpha}) .
\end{aligned}
$$

Here we have used the identity

$$
\boldsymbol{\alpha}^{T}\left(E \cdot \mathbf{l}^{*}(\boldsymbol{\alpha})\right)=\boldsymbol{\alpha}^{T}\left\langle\left(E \cdot \nabla_{v} \mathbf{m}\right) \mathcal{M}(\boldsymbol{\alpha})\right\rangle=\frac{m_{e}}{\theta_{\ell}} E \cdot\langle v \mathcal{M}(\boldsymbol{\alpha})\rangle ;
$$

this follows from definition (2.7) of $l^{*}$ and the fact that $\left\langle E \cdot \nabla_{\nu} \eta^{*}\right\rangle=0$, where by (1.11) and (2.4) one has $E \cdot \nabla_{\nu} \eta^{*}=\left(E \cdot \nabla_{\nu} \mathbf{m}\right) \mathcal{M}(\boldsymbol{\alpha})-\frac{m_{e}}{\theta_{\ell}} E \cdot \nu \mathcal{M}(\boldsymbol{\alpha})$.

The right side of (2.12) has the form

$$
\begin{aligned}
\boldsymbol{\alpha}^{T} \mathbf{r}^{*}(\boldsymbol{\alpha})=\left\langle\boldsymbol{\alpha}^{T} \mathbf{m} C(\mathcal{M}(\boldsymbol{\alpha}))\right\rangle \\
=\left\langle\partial_{F} \eta(\mathcal{M}(\boldsymbol{\alpha})) C(\mathcal{M}(\boldsymbol{\alpha}))\right\rangle,
\end{aligned}
$$

which by the local dissipation relation (1.5) for the collision operator $C$ then implies that

$$
\boldsymbol{\alpha}^{T} \mathbf{r}^{*}(\boldsymbol{\alpha}) \leq 0
$$

This establishes the dissipative character of (2.12). Moreover, the characterization (1.6) of the equilibria of $C$ shows that equilibria of $r^{*}$ are analogously characterized by the equivalence of the following statements:

(i) $\boldsymbol{\alpha}^{T} \mathbf{r}^{*}(\boldsymbol{\alpha})=0, \quad$ (ii) $\mathbf{r}^{*}(\boldsymbol{\alpha})=0$,

(iii) $\mathcal{M}(\boldsymbol{\alpha})$ is an equilibrium of the form (1.12).

Finally, by using (1.10), the electron entropy density and flux that appear in (2.12) may be put into the form

$$
\begin{aligned}
\boldsymbol{\alpha}^{T} h_{\boldsymbol{\alpha}}^{*}(\boldsymbol{\alpha})-h^{*}(\boldsymbol{\alpha}) & =\left\langle\boldsymbol{\alpha}^{T} \mathbf{m} \mathcal{M}(\boldsymbol{\alpha})\right\rangle-\left\langle\eta^{*}\left(\boldsymbol{\alpha}^{T} \mathbf{m}\right)\right\rangle \\
& =\langle\eta(\mathcal{M}(\boldsymbol{\alpha}))\rangle, \\
\boldsymbol{\alpha}^{T} j_{\boldsymbol{\alpha}}^{*}(\boldsymbol{\alpha})-j^{*}(\boldsymbol{\alpha}) & =\left\langle v \boldsymbol{\alpha}^{T} \mathbf{m} \mathcal{M}(\boldsymbol{\alpha})\right\rangle-\left\langle\eta^{*}\left(\boldsymbol{\alpha}^{T} \mathbf{m}\right)\right\rangle \\
& =\langle\nu \eta(\mathcal{M}(\boldsymbol{\alpha}))\rangle .
\end{aligned}
$$

It is seen from (2.15) and (2.18) that the local dissipation law (2.12) for the moment system is exactly the local dissipation law (1.9) for the Boltzmann-Poisson system evaluated at $F=\mathbf{M}(\boldsymbol{\alpha})$. This entropy structure implies [5] that, when expressed in terms of the 
entropy densities, each moment system (2.5) fits into the framework of "extended thermodynamics" [7] and, more generally, relaxation systems [1].

\section{Acknowledgements}

This work was supported by the NSF under grant DMS-9404570 at the University of Arizona. Some of this work was carried out while the author was visiting the Institute for Advanced Study under a grant from the Alfred P. Sloan Foundation.

\section{References}

[1] G.Q. Chen, C.D. Levermore and T.P. Liu, Hyperbolic Conservation Laws with Stiff Relaxation Terms and Entropy. Comm. Pure \& Appl. Math. 47 (1994), 787-830.

[2] S.K. Godunov, An Interesting Class of Quasilinear Systems, Sov. Math. Dokl. 2 (1961), 947-949; original Russian, DAN. USSR 139 (1961) 521-523.
[3] H. Grad, On the Kinetic Theory of Rarefied Gases, Comm. Pure \& Appl. Math. 2 (1949) 331-407.

[4] C.D. Levermore, Moment Closure Hierarchies for Kinetic Theories, J. Stat. Phys. 83 (1996) 1021-1065.

[5] C.D. Levermore, Entropy-Based Moment Closure Hierarchies for the Boltzmann-Poisson Equation, Phys. Rev. B (submitted 1998).

[6] P.A. Markowich, C.A. Ringhofer and C. Schmeiser, Semiconductor Equations, Springer-Verlag, Wein (1990).

[7] I. Müller and T. Ruggeri, Extended Thermodynamics, Springer Tracts in Nat. Phil. 37, Springer-Verlag, New York (1993).

\section{Biography}

Professor Levermore has been a member of the Department of Mathematics at the University of Arizona since 1988. His interests include partial differential equations, mathematical physics, integrable and near integrable systems, and numerical analysis. 

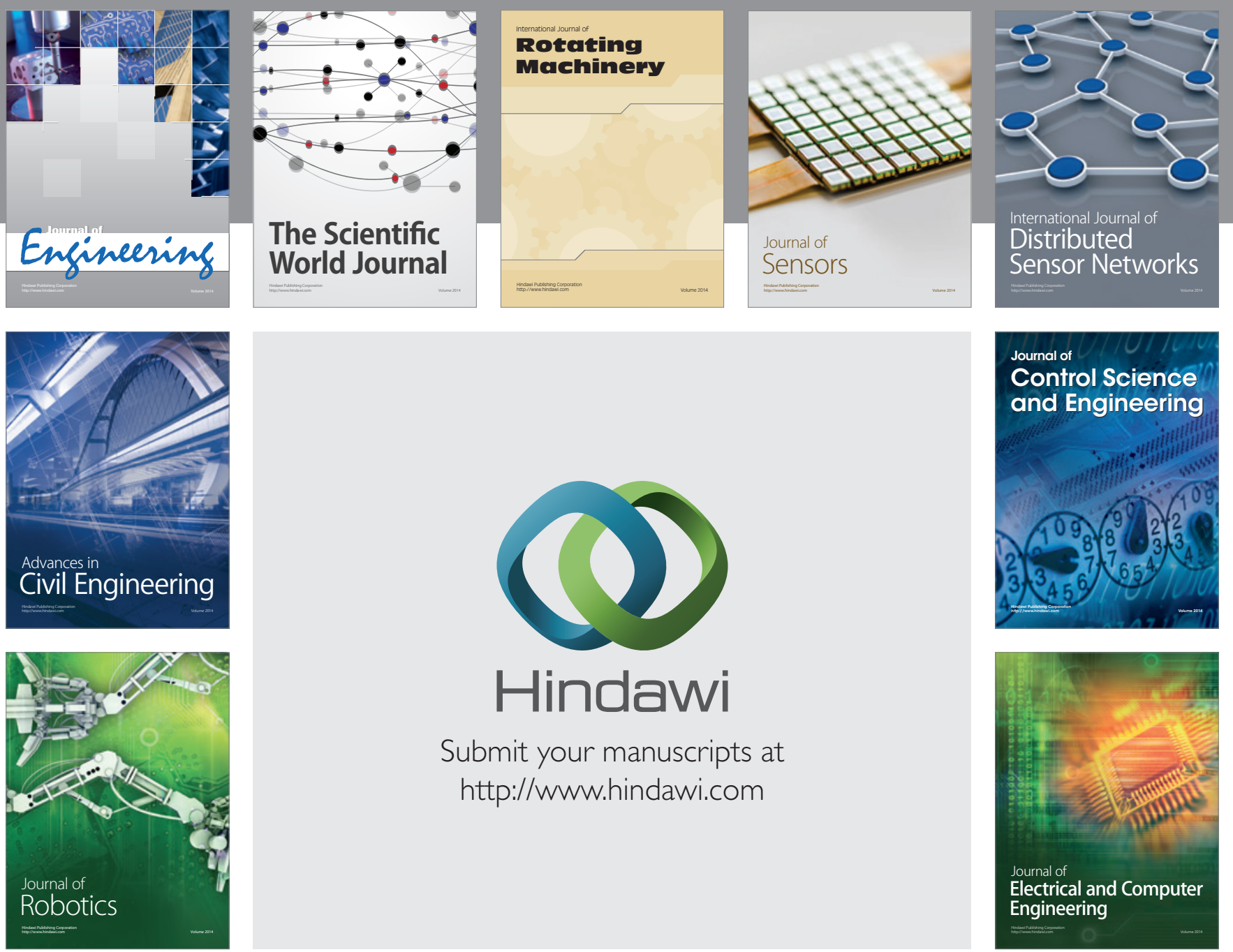

Submit your manuscripts at

http://www.hindawi.com
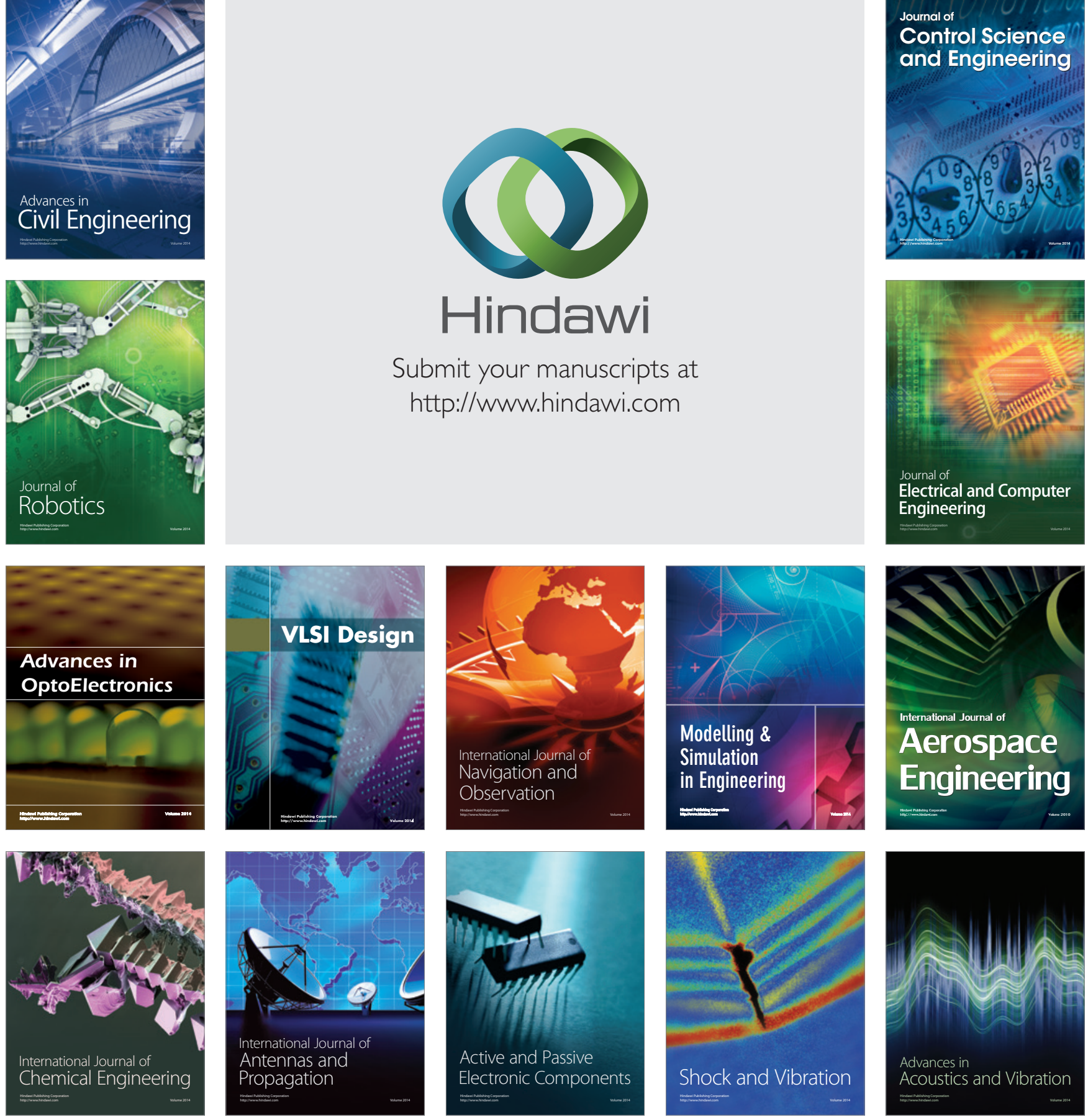